\section{(6) OPEN ACCESS}

\title{
Anti-inflammatory treatment improves high-density lipoprotein function in rheumatoid arthritis
}

\author{
Francis O'Neill, ${ }^{1}$ Marietta Charakida, ${ }^{1}$ Eric Topham, ${ }^{2}$ Eve McLoughlin, ${ }^{1}$ Neha Patel, \\ Emma Sutill, ${ }^{1}$ Christopher W M Kay, ${ }^{2}$ Francesco D'Aiuto, ${ }^{3}$ Ulf Landmesser, ${ }^{4}$ \\ Peter C Taylor, ${ }^{5}$ John Deanfield ${ }^{1,6}$
}

\begin{abstract}
- Additional material is published online only. To view please visit the journal online (http://dx.doi.org/10.1136/ heartjnl-2015-308953).
\end{abstract}

For numbered affiliations see end of article.

\section{Correspondence to} Professor John Deanfield, National Centre for

Cardiovascular Prevention and Outcomes (incorporating NICOR), UCL Institute of

Cardiovascular Sciences,

Nomura House-Level 2 East,

1 St Martin's Le Grand, London EC1A 4NP, UK:

j.deanfield@ucl.ac.uk

Preliminary results from this study were presented at the European Society of Cardiology Congress in 2014 (abstract no. 87557).

Received 11 November 2015 Revised 20 September 2016 Accepted 12 October 2016

Published Online First

16 November 2016

\begin{abstract}
Objective Patients with rheumatoid arthritis (RA) are at increased cardiovascular risk. Recent studies suggest that high-density lipoprotein (HDL) may lose its protective vascular phenotype in inflammatory conditions. However, the effects of common antiinflammatory treatments on HDL function are not yet known.

Methods We compared the function of HDL in 18 patients with RA and 18 matched healthy controls. Subsequently, patients were randomised to (methotrexate +infliximab $(\mathrm{M}+\mathrm{l})(5 \mathrm{mg} / \mathrm{kg}))$ or methotrexate+placebo $(\mathrm{M}+\mathrm{P})$ infusions for 54 weeks. At week 54 and thereafter, all patients received infliximab therapy until completion of the trial (110 weeks), enabling assessment of the impact of 1 year of infliximab therapy in all patients. HDL functional properties were assessed at baseline, 54 weeks and 110 weeks by measuring the impact on endothelial nitric oxide (NO) bioavailability and superoxide production (SO), paraoxonase activity (PON-1) and cholesterol efflux.
\end{abstract}

Results All HDL vascular assays were impaired in patients compared with controls. After 54 weeks, NO in response to $\mathrm{HDL}$ was significantly greater in patients who received $\mathrm{M}+\mathrm{I}$ compared with those who received $M+P$. Endothelial SO in response to HDL was reduced in both groups, but PON-1 and cholesterol efflux remained unchanged. All vascular measures improved compared with baseline after $\geq 1$ infliximab therapy in the analysis at 110 weeks. No significant trend was noted for cholesterol efflux.

Conclusions HDL function can be improved with antiinflammatory treatment in patients with RA. The M+l combination was superior to the $M+P$ alone, suggesting that the tumour necrosis factor- $\alpha$ pathway may have a role in HDL vascular properties.

\section{INTRODUCTION}

Cardiovascular (CV) disease remains the leading cause of morbidity and mortality in patients with rheumatoid arthritis (RA). ${ }^{1}$ Conventional risk factors do not account fully for this, and increased levels of inflammation associated with RA may be an important determinant of $\mathrm{CV}$ outcomes. ${ }^{2}$ In a study of 651 patients, an elevated inflammatory state was associated with an increased CV event rate but a paradoxical reduction in circulating lipid levels. ${ }^{3}$ This suggests that the relationship between lipid levels and increased CV disease is altered in RA and may be explained, at least in part, by qualitative changes to lipoproteins as a result of the inflammatory milieu.

High-density lipoprotein (HDL) has been suggested to exert anti-atherosclerotic effects via reverse cholesterol transport and activation of protective endothelial pathways. In conditions such as RA however, HDL has been shown to acquire pro-inflammatory and pro-thrombotic phenotype that can promote atherogenesis and potentially increase CV risk. ${ }^{4}{ }^{5}$ A previous study from our group demonstrated that the beneficial function of HDL can be restored after resolution of acute inflammatory stimulus, suggesting that the modification of systemic inflammation offers potential for $\mathrm{CV}$ risk reduction. ${ }^{6}$

In our current study, we examined the influence of standard (methotrexate (MTX)) and novel antiinflammatory treatments (infliximab) on HDL function in patients with RA in a randomised controlled trial over 1 year. We also assessed the long-term effect of infliximab on HDL function and CV and RA risk factors. Our findings support the concept that HDL dysfunction can be improved by antiinflammatory drugs that are widely used for RA therapy and this may confer $\mathrm{CV}$ benefit.

\section{METHODS}

\section{Study population and protocol}

RA population

We studied 18 patients with early erosive RA who were required to have (i) a diagnosis of RA according to the American College of Rheumatology 1987 criteria, (ii) symptoms for 6 months-3 years, (iii) a minimum of two swollen metacarpophalangeal (MCP) joints despite treatment with MTX and (iv) seropositivity for IgM rheumatoid factor. In addition, eligible patients were required to have either (i) erosion of $\geq 1 \mathrm{MCP}$ joint as demonstrated on plain radiography or as a cortical break with irregular margins (or contour) on greyscale ultrasound in both the longitudinal and transverse scanning planes or (ii) erosions of $\geq 2 \mathrm{MCP}$ joints (cortical breaks with irregular margins/contour on greyscale ultrasound in either the transverse or the longitudinal plane associated with a strong vascular signal in power Doppler mode at the site of the cortical break). ${ }^{7}$

All patients received oral MTX for greater than or equal to at least 8 weeks, at a minimum stable dosage of $12.5 \mathrm{mg} /$ week but not exceeding $17.5 \mathrm{mg} /$ week. Patients being treated with oral
Charakida M, Topham E,

766-773. 
corticosteroids must have been receiving a stable dosage (10 mg prednisolone per day) for 4 weeks.

Study 1: case-control study

Vascular properties of HDL from 18 patients with RA were compared with 18 healthy control subjects. Healthy controls had no CV risk factors by history, clinical examination and laboratory tests, and were matched to patients with RA for age and gender.

\section{Study 2: randomised clinical trial (double-blind phase)-} secondary analysis

All physicians, patients, nurses and other non-clinical members of the study team were blinded for the first year of the study. Eighteen patients with RA were randomised into one of two treatment groups by a pharmacist who did not participate in the screening visit. Eleven patients received infusions of infliximab at $5 \mathrm{mg} / \mathrm{kg}$ and seven received placebo (normal saline) infusions at weeks 0,2 and 6, and then every 8 weeks through week 46. At the end of the first year, all patients were maintained a singleblinded study for a further year. Patients in the methotrexate + infliximab $(\mathrm{M}+\mathrm{I})$ group received infliximab infusions at weeks $54,56,62$ and thereafter every 8 weeks. Those in the $\mathrm{M}+\mathrm{I}$ group received a placebo infusion at week 56 in order to maintain blinding and received infliximab infusions every 8 weeks until the end of the study (110 weeks) (see online supplementary appendix figure 1). As a result, all patients received a minimum of 1 year of infliximab in the two phases of the study.

Baseline dosages of MTX or corticosteroid were maintained during the first 18 weeks of the study. After week 18, if any patient failed to achieve a 50\% reduction from baseline in the number of swollen hand and wrist joints, the weekly dose of MTX was increased by $2.5 \mathrm{mg}$ once every 4 weeks until a $50 \%$ reduction from baseline in the number of swollen hand and wrist joints was achieved, until the dosage of oral MTX reached $25 \mathrm{mg} /$ week or until the dose escalation was limited by toxicity. Thereafter, irrespective of their response status, patients continued to receive the maximum tolerated MTX dosage until the end of the study. The study was approved by Riverside Research Ethics Committee, and all patients provided informed consent. Clinical monitoring of the study was performed independently (Centocor, Malvern, Pennsylvania, USA).

\section{Laboratory assays}

Anthropometric and biochemical measurements

Anthropometric measurements were made and body mass index (BMI $\left(\mathrm{kg} / \mathrm{m}^{2}\right)$ ) was calculated from weight and height. Blood pressure was measured in triplicate (HEM-705CP, Omron), and the average of the readings was calculated. Blood was drawn and processed after an overnight fast, and serum and plasma samples were stored at $-70^{\circ} \mathrm{C}$ for subsequent analysis. Full blood count, lipid and glucose level measurements were made with standard biochemistry assays and $\mathrm{C}$ reactive protein (CRP) was measured with an immunoturbidimetric, high-sensitivity assay (Tina-quant assay performed on a Cobas Integra analyzer, Roche Diagnostics).

\section{HDL measurements}

Measurements of HDL function were carried out at baseline, 54 weeks and 110 weeks in patients with RA. Baseline measurements were compared with those from healthy controls.

\section{HDL isolation}

HDL was isolated by sequential ultracentrifugation $(\mathrm{d}=1.063-$ $1.21 \mathrm{~g} / \mathrm{mL}$ ) using solid potassium bromide for density adjustment. $^{8}$ All functional assays of HDL were carried out within two weeks of isolation by a blinded investigator in duplicate.

\section{Endothelial nitric oxide bioavailability}

The effect of HDL $\left(50 \mu \mathrm{g} / \mathrm{mL}: 60 \mathrm{~min}, 37^{\circ} \mathrm{C}\right)$ on endothelial nitric oxide (NO) bioavailability (bovine aortic endothelial cells (BAECs): passage 4-7; Lonza Bioscience) was measured using a fluorescent indicator. BAECs were incubated with 4,5-diaminofluorescein diacetate (DAF-2; 1Um; Cayman Chemical), and triazolofluorescein fluorescence was measured using an excitation wavelength of $485 \mathrm{~nm} .^{9}$

\section{Endothelial superoxide production}

The effect of HDL on endothelial cell superoxide production (SO) was measured in unstimulated and tumour necrosis factor- $\alpha$ (TNF- $\alpha$ )-stimulated $(5 \mathrm{ng} / \mathrm{mL}, \mathrm{R} \& \mathrm{D}$ Systems) human aortic endothelial cells (HAECs) by erythrocyte sedimentation rate (ESR) spectroscopy. HAECs were incubated with HDL from patients and controls $\left(50 \mu \mathrm{g} / \mathrm{mL}, 60 \mathrm{~min}, 37^{\circ} \mathrm{C}\right)$, with or without TNF- $\alpha$ and resuspended in Krebs-Hepes buffer (pH 7.4; Noxygen) containing diethyldithiocarbamic acid sodium salt (5 $\mu \mathrm{M}$, Noxygen) and deferoxamine methanesulfonate salt ( $25 \mu \mathrm{M}$, Noxygen). ESR spectra were recorded after addition of the spin probe 1-hydroxy-3-methoxycarbonyl-2,2,5,5-tetramethylpyrrolidine $(\mathrm{CMH}$; Noxygen; final concentration $200 \mu \mathrm{M}$ ) using a Bruker e-scan spectrometer (Bruker Biospin). The ESR instrumental settings were centre field (B0) $3495 \mathrm{G}$; field sweep width $10 \mathrm{G}$; microwave frequency $9.75 \mathrm{GHz}$; microwave power $19.91 \mathrm{~mW}$; magnetic field modulation frequency $86.00 \mathrm{kHz}$; modulation amplitude $2.60 \mathrm{G}$; conversion time $10.24 \mathrm{~ms}$; and number of $\mathrm{x}$-scans $1020 .^{9}$

\section{Cholesterol efflux capacity}

HDL for measurements of efflux capacity was extracted from serum by ApoB depletion. Whole serum was incubated for 20 min with a $20 \%$ polyethylene glycol (PEG) solution $(20 \%$ PEG 8000 (sigma P2139) in $200 \mathrm{mM}$ glycine (sigma G8898, $\mathrm{pH}=10)$ ). Samples were centrifuged at $1900 \mathrm{~g}$, and the supernatant was collected and stored at $4^{\circ} \mathrm{C}$. J774 cells were radiolabeled for 24 hours in a medium containing $2 \mu \mathrm{Ci}$ of $[3 \mathrm{H}]$-cholesterol per microlitre. Addition of $0.3 \mathrm{mM} 8$-(4-chlorophenylthio)-cyclic AMP for 6 hours upregulated expression of ABCA1. An efflux medium containing 2.8\% apolipoprotein B-depleted serum from each individual was added for 4 hours. To prevent cholesterol esterification during labelling, equilibration and flux, $2 \mu \mathrm{g} / \mathrm{mL}$ of $\mathrm{CP} 113,818$, a acyl-coenzyme A:cholesterol acyltransferase inhibitor was added to all mediums. Efflux capacity was quantified using liquid scintillation to measure radioactive cholesterol effluxed from the cells (medium+intracellular lipids). All assays were performed in duplicate and the final average value normalised against a baseline control for statistical analyses between time points. $^{10}$

\section{Paraoxonase-1 activity}

Serum paraoxonase activities were measured by ultraviolet spectrophotometry in a 96-well plate format using paraoxon (Sigma-Aldrich, St Louis, Missouri, USA). Briefly, $50 \mu \mathrm{g} / \mathrm{mL}$ HDL was diluted in a reaction mixture containing $10 \mathrm{mM}$ Tris hydrochloride ( $\mathrm{pH} 8.0$ ), $1 \mathrm{M}$ sodium chloride and $2 \mathrm{mM}$ calcium chloride. At $24^{\circ} \mathrm{C}, 1.5 \mathrm{mM}$ paraoxon was added to initiate the reaction, and the increase in absorbance at $405 \mathrm{~nm}$ was recorded 
over $30 \mathrm{~min}$. An extinction coefficient (at $405 \mathrm{~nm}$ ) of 17000 $\mathrm{M}^{-1} \cdot \mathrm{cm}^{-1}$ was used to calculate units of paraoxonase-1 (PON-1). ${ }^{11}$

\section{Statistics}

HDL studies were powered for a 1:1 randomised controlled trial on the basis of NO bioavailability using paired assessments in 35 healthy controls (mean $=0.98, \mathrm{SD}=0.13$, and intraclass correlation 0.91). ${ }^{12}$ From this power calculation, a total of seven patients per group was required (power $=80 \%$ and $\alpha=0.05$ ) to detect a $5 \%$ difference in the primary outcome (NO bioavailability). Normal distribution was assessed using the Shapiro-Wilk test. All measures are reported as mean (SD) or median (IQR) for those not normally distributed. Baseline comparisons were performed using an independent t-test or Mann-Whitney U test if data was non-normally distributed.

Post hoc multivariate analysis of variance (ANOVA) modelling was performed to evaluate the effects of treatment with infliximab (vs placebo) and duration of treatment on HDL function, $\mathrm{CV}$ and clinical markers at 54 weeks. A repeated-measures ANOVA, with time point comparisons using Bonferronicorrected t-tests, was performed to determine differences in HDL function, CV and clinical markers in patients who received 2 years infliximab treatment. The Greenhouse-Geisser correction for the $\mathrm{F}$ test was used to adjust the degrees of freedom for deviation from sphericity. Analysis was performed using GraphPad Prism analysis software. A two-sided $p$ value of $<0.05$ was considered to indicate statistical significance. An additional multilevel logistic regression analysis was conducted in $\mathrm{R}$ to confirm our results (see online supplementary appendix 2). We fit multilevel logistic regression models for measure of HDL function using the lme4 package (http://cran.r-project.org/web/ packages/lme4/index.html) in the $\mathrm{R}$ statistical language (http:// www.r-project.org/). All coefficients were taken as random effects.

Table 1 Baseline characteristics in rheumatoid arthritis and healthy participants

\begin{tabular}{|c|c|c|c|}
\hline & $\begin{array}{l}\text { Control } \\
n=18\end{array}$ & $\begin{array}{l}\text { Rheumatoid } \\
\text { arthritis } \\
n=18\end{array}$ & p Value \\
\hline \multicolumn{4}{|l|}{ Demographics } \\
\hline Age (years) & $55.61(6.37)$ & $58.56(11.05)$ & 0.20 \\
\hline $\begin{array}{l}\text { Gender (proportion } \\
\text { female) }\end{array}$ & $10 / 18$ & $12 / 18$ & 0.48 ( $\chi^{2}$ test) \\
\hline \multicolumn{4}{|l|}{ Cardiovascular risk } \\
\hline BMI $\left(k g / m^{2}\right)$ & $26.27(2.29)$ & $24.42(4.71)$ & 0.14 \\
\hline $\begin{array}{l}\text { Total cholesterol } \\
\text { (mmol/L) }\end{array}$ & $4.93(0.66)$ & $5.00(1.43)$ & 0.79 \\
\hline $\mathrm{HDL}(\mathrm{mmol} / \mathrm{L})$ & $1.35(0.44)$ & $1.02(0.19)$ & 0.01 \\
\hline $\mathrm{LDL}(\mathrm{mmol} / \mathrm{L})$ & $2.89(0.88)$ & $3.01(1.19)$ & 0.74 \\
\hline $\begin{array}{l}\text { Triglyceride } \\
\text { (mmol/L) }\end{array}$ & $1.13(0.36)$ & $2.19(0.96)$ & $<0.01$ \\
\hline Glucose (mg/dL) & $4.95[4.70-5.82]$ & $4.49[3.46-4.94]$ & 0.01 \\
\hline \multicolumn{4}{|l|}{ Inflammation } \\
\hline CRP (mg/L) & 1.00 [1.00-1.00] & 22.74 [13.76-40.78] & $<0.01$ \\
\hline \multicolumn{4}{|c|}{$\begin{array}{l}\text { Values expressed as mean (SD) and median [IQR] for non-normally distributed data. } \\
\text { Comparisons between rheumatoid patients and healthy controls were performed } \\
\text { using the independent t-test. Categorical variables were compared using Fisher's } \\
\text { exact } \chi^{2} \text { test. Comparisons for non-parametric measurements were performed using } \\
\text { Mann-Whitney test. } \\
\text { BMI, body mass index; CRP, C reactive protein; HDL, high-density lipoprotein; LDL, } \\
\text { low-density lipoprotein. }\end{array}$} \\
\hline
\end{tabular}

RESULTS

Cross-sectional study: comparison between patients with RA and controls

The patients and controls were well matched for age, gender and BMI (table 1). Patients with RA had significantly lower HDL-cholesterol (HDL-c) levels $(p=0.01)$, but increased triglyceride levels $(\mathrm{p}<0.01)$. Total cholesterol and low-density lipoprotein (LDL)-c were similar between the groups (table 1). Patients with RA had significantly higher CRP levels $(\mathrm{p}<0.01)$ and lower blood glucose levels compared with controls $(\mathrm{p}=0.01)$.

HDL, which was isolated from patients with RA, showed reduced NO bioavailability and increased SO production $(\mathrm{p}<0.01$ for both) in cultured endothelial cells compared with controls. Paraoxonase activity was also impaired in patients with RA $(p=0.03)$ (figure 1).

Double-blind randomised control study: lipid levels and HDL functional properties after 1 year of treatment

Both treatment groups were balanced for CV risk factors, RA disease activity and HDL function at baseline (table 2). An increase in total cholesterol levels was observed in the

A

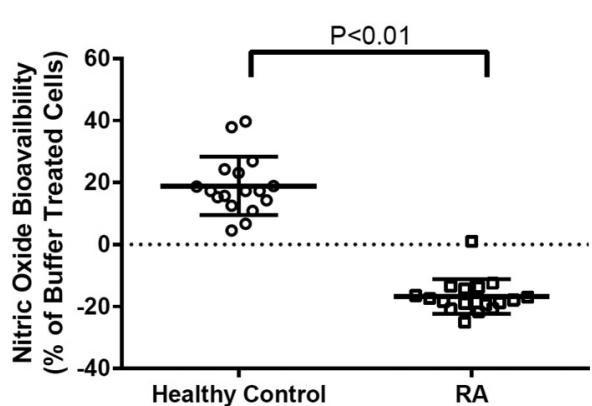

B

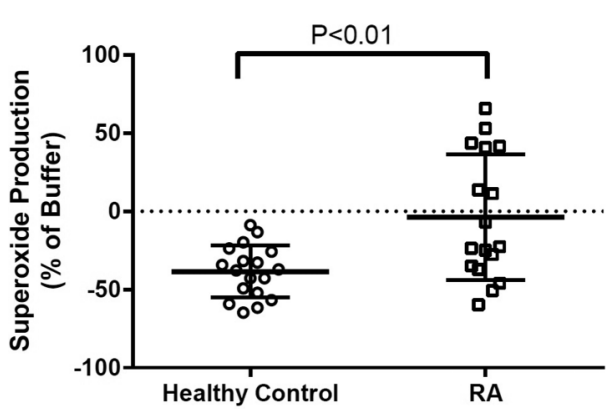

C

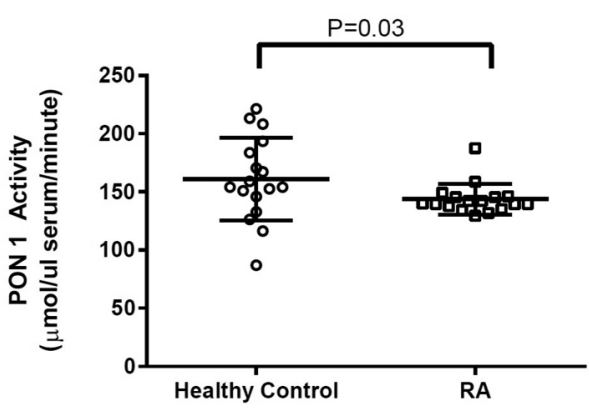

Figure 1 Comparison of the functional properties of high-density lipoprotein between patients with rheumatoid arthritis (RA) and healthy controls. (A) Nitric oxide bioavailability. (B) Superoxide production. (C) Paraoxonase-1 (PON-1) activity. Individual data points for each patients $(n=18$ in each group). Error bars represent mean and SEM. 
Table 2 Baseline characteristics of patients with RA entering randomised trial

\begin{tabular}{|c|c|c|c|}
\hline & $\begin{array}{l}\text { Placebo } \\
n=7\end{array}$ & $\begin{array}{l}\text { Infliximab } \\
\mathrm{n}=11\end{array}$ & $\mathrm{p}$ Value \\
\hline \multicolumn{4}{|l|}{ Demographics } \\
\hline Age (years) & $55.86(15.89)$ & $61.30(11.05)$ & 0.42 \\
\hline Gender (proportion female) & $4 / 7$ & $8 / 11$ & 0.31 ( $\chi^{2}$ test $)$ \\
\hline \multicolumn{4}{|l|}{ Cardiovascular risk } \\
\hline $\mathrm{BMI}\left(\mathrm{kg} / \mathrm{m}^{2}\right)$ & $25.88(0.55)$ & $24.98(5.11)$ & 0.73 \\
\hline Total cholesterol (mmol/L) & $5.05(1.42)$ & $5.01(1.5)$ & 0.96 \\
\hline $\mathrm{HDL}-\mathrm{c}(\mathrm{mmol} / \mathrm{L})$ & $1.10(0.22)$ & $0.97(0.17)$ & 0.20 \\
\hline LDL-c (mmol/L) & $3.20(0.95)$ & $2.90(1.34)$ & 0.62 \\
\hline Triglyceride (mmol/L) & $1.67(1.00)$ & $2.52(0.81)$ & 0.63 \\
\hline Glucose (mg/dL) & $4.60(0.70)$ & $4.75(1.24)$ & 0.59 \\
\hline Systolic BP (mm Hg) & $140.70(7.27)$ & $132.5(4.67)$ & 0.33 \\
\hline Diastolic BP (mm Hg) & $75.71(3.96)$ & $76.00(3.70)$ & 0.96 \\
\hline \multicolumn{4}{|l|}{ HDL function } \\
\hline Nitric oxide bioavailability (\% buffer-treated cells) & $81.81(3.97)$ & $84.23(6.23)$ & 0.37 \\
\hline Superoxide production (nmol/ $/ \mathrm{O}_{2} / 100000$ cells) & $4.37(1.71)$ & $3.90(1.66)$ & 0.56 \\
\hline Paraoxonase-1 activity ( $\mu \mathrm{mol}$ p-nitrophenol/L/serum/min) & $137.52(1.71)$ & $148.24(14.49)$ & 0.09 \\
\hline Efflux capacity (\%) & $12.25(2.04)$ & $13.52(1.26)$ & 0.31 \\
\hline \multicolumn{4}{|l|}{ RA disease activity } \\
\hline CRP (mg/L) & $37.65[19.48-64.31]$ & $26.83[14.37-37.33]$ & 0.22 \\
\hline ESR (mm/hours) & $30.00[22.00-76.00]$ & $25.00[14.00-50.50]$ & 0.36 \\
\hline DAS28-CRP score & $6.08(0.71)$ & $5.17(1.13)$ & 0.09 \\
\hline DAS28-ESR score & $5.59(0.85)$ & $4.68(1.56)$ & 0.15 \\
\hline
\end{tabular}

Table 3 Clinical characteristics and HDL function at 1 year

\begin{tabular}{|c|c|c|c|c|c|c|}
\hline & \multicolumn{3}{|l|}{ MTX+placebo } & \multicolumn{3}{|l|}{ MTX+infliximab } \\
\hline & Baseline & One year & $p$ Value & Baseline & One year & p Value \\
\hline \multicolumn{7}{|l|}{ Cardiovascular risk } \\
\hline Total cholesterol (mmol/L) & $5.05(1.42)$ & $5.13(1.55)$ & 0.84 & $5.01(1.5)$ & $6.23(1.63)$ & 0.04 \\
\hline HDL-c (mmol/L) & $1.10(0.22)$ & $1.10(0.42)$ & 0.14 & 1.02 [0.93-1.09] & $1.33[1.26-1.50]$ & 0.02 \\
\hline LDL-c (mmol/L) & $3.20(0.95)$ & $3.18(1.23)$ & 0.96 & $2.90(1.34)$ & $3.65(1.22)$ & 0.07 \\
\hline Triglyceride (mmol/L) & 1.35 [1.07-1.62] & 1.26 [1.12-1.43] & 0.20 & $2.52(0.81)$ & $2.48(0.92)$ & 0.91 \\
\hline Glucose (mg/dL) & $4.59[4.28-4.76]$ & $4.40[4.04-5.12]$ & 0.61 & $4.75(1.24)$ & $4.99(0.95)$ & 0.15 \\
\hline \multicolumn{7}{|l|}{ HDL function } \\
\hline $\begin{array}{l}\text { Nitric oxide bioavailability } \\
\text { (\% buffer-treated cells) }\end{array}$ & $81.81(3.97)$ & $82.72(3.43)$ & 0.67 & $84.23(6.23)$ & $89.08(9.31)$ & 0.02 \\
\hline $\begin{array}{l}\text { Superoxide production } \\
\text { (nmol/ } / 0_{2} / 100000 \text { cells) }\end{array}$ & $4.37(1.71)$ & $3.63(1.67)$ & 0.03 & $3.90(1.66)$ & $2.98(1.47)$ & 0.01 \\
\hline $\begin{array}{l}\text { Paraoxonase-1 activity } \\
\text { ( } \mu \mathrm{mol} \text { p-nitrophenol/L/serum/min) }\end{array}$ & $137.52(6.41)$ & $153.58(17.88)$ & 0.07 & $148.24(14.49)$ & $155.15(14.86)$ & 0.07 \\
\hline Efflux capacity (\%) & $12.25(2.04)$ & $13.25(2.52)$ & 0.26 & $13.52(1.26)$ & $14.01(3.23)$ & 0.72 \\
\hline \multicolumn{7}{|l|}{ RA disease activity } \\
\hline CRP (mg/L) & 37.65 [19.48-64.31] & 26.00 [13.85-36.83] & 0.09 & 26.83 [14.37-37.33] & $2.34[0.00-6.34]$ & 0.03 \\
\hline ESR (mm/hours) & $30.00[22.00-76.00]$ & 27.50 [16.00-38.00] & 0.69 & $25.00[14.00-50.50]$ & $20.50[11.00-30.00]$ & 0.61 \\
\hline DAS28-CRP score & $6.08(0.71)$ & $5.04(0.85)$ & 0.14 & $5.17(1.13)$ & $3.53(1.06)$ & $<0.01$ \\
\hline DAS28-ESR score & $5.59(0.85)$ & $4.43(1.74)$ & 0.08 & $4.68(1.56)$ & $2.25(0.83)$ & $<0.01$ \\
\hline
\end{tabular}

Values expressed as mean (SD) and median [IQR] for non-normally distributed data. Comparisons between groups were performed using a paired t-test. Comparisons for non-parametric measurements were performed using Wilcoxon signed-rank test.

CRP, C reactive protein; ESR, erythrocyte sedimentation rate; HDL-c, high-density lipoprotein cholesterol; LDL-c, low-density lipoprotein cholesterol; MTX, methotrexate; RA, rheumatoid arthritis.

infliximab arm at 54 weeks $(\mathrm{p}=0.04)$ and was primarily driven by an increase of HDL-c $(p=0.02) . M+P$ treatment did not alter lipid profile. CRP was significantly reduced with infliximab treatment compared with MTX alone $(p=0.03)$, which did not demonstrate a significant change at 54 weeks. Clinical and laboratory measures of RA were improved with $\mathrm{M}+\mathrm{I}$ therapy, but no changes were detected in the $\mathrm{M}+\mathrm{P}$ arm (table 3). 


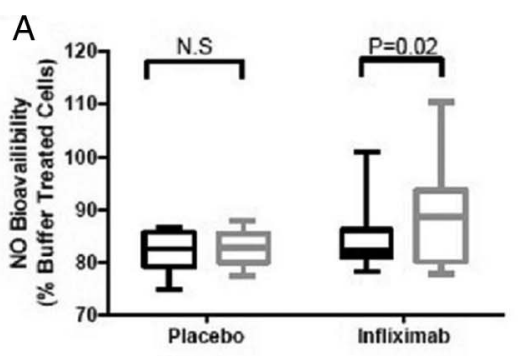

C

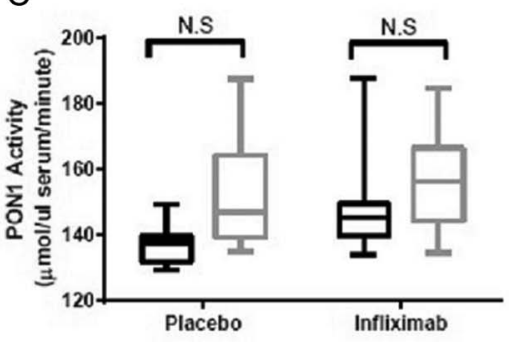

B
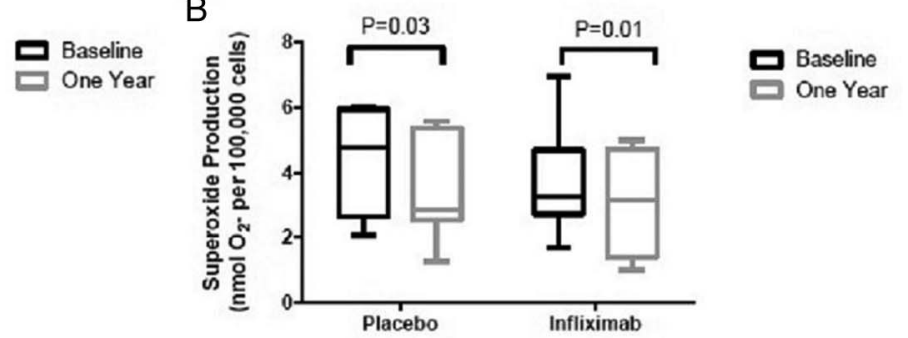

$\mathrm{D}$

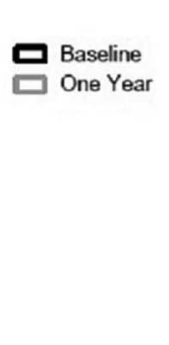

\section{送}

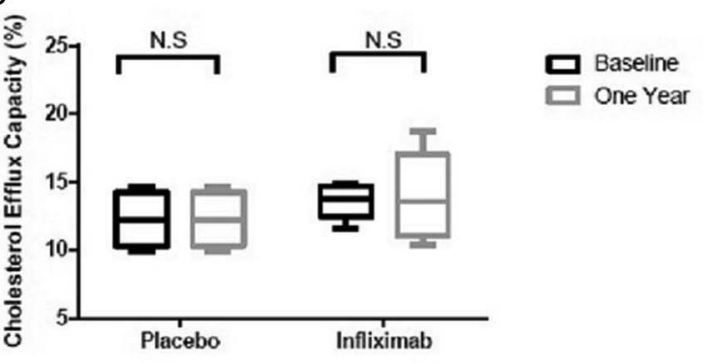

Figure 2 Comparison of (A) nitric oxide (NO) bioavailability, (B) superoxide production, (C) paraoxonase-1 (PON-1) activity and (D) cholesterol efflux during placebo controlled phase of trial. Box and whisker plot represents mean and $95 \% \mathrm{Cls}$.

SO production was reduced from baseline following both $\mathrm{M}+\mathrm{P}$ and $\mathrm{M}+\mathrm{I}$ treatments $(\mathrm{p}=0.03$ and 0.01$)$. NO bioavailability was improved $(p=0.02)$ with $M+I$ therapy, but no effect was noted in the $\mathrm{M}+\mathrm{P}$ patients. PON-1 activity increased in a non-significant manner in both groups, but cholesterol efflux capacity remained unchanged (figure 2). Between-treatment comparisons at 54 weeks did not demonstrate benefit of $\mathrm{M}+\mathrm{I}$ treatment over $\mathrm{M}+\mathrm{P}$ treatment for any measure of HDL function. These results were confirmed in a multilevel logistic regression model (see online supplementary appendix 2).

\section{Single-blinded study}

In grouped analysis of the 18 patients who received 54 weeks of $\mathrm{M}+\mathrm{I}$ treatment, NO bioavailability increased compared with baseline $(p<0.01)$. Furthermore, a reduction in SO $(p<0.01)$ and an increase of PON-1 (both $\mathrm{p}=0.03$ ) was seen (figure 3). No change in cholesterol efflux capacity was noted. Lipid levels were increased and inflammatory and RA disease activity scores were significantly reduced (data not shown).

\section{Two years infliximab treatment}

Eleven patients received infliximab therapy for 110 weeks. Total cholesterol $(p=0.08)$ and LDL-c $(p=0.09)$ showed a nonsignificant increase at 110 weeks, but there was a significant increase in HDL-c $(p=0.01)$. CRP and RA disease activity scores were both significantly reduced. No changes were noted for cholesterol efflux (figure 4).

Repeated-measures ANOVA demonstrated a significant increase in NO bioavailability $(p=0.01)$ and significant reductions in SO production $(p=0.02)$ and PON-1 $(p=0.01)$. There was no further improvement in HDL function between patients who had received 54 weeks of $\mathrm{M}+\mathrm{I}$ treatment compared with 110 weeks of $\mathrm{M}+\mathrm{I}$ treatment. All measures of HDL function remained impaired compared with the healthy controls (data not shown).

\section{DISCUSSION}

This study shows that anti-inflammatory treatments can improve the antioxidant and endothelial protective properties of HDL in
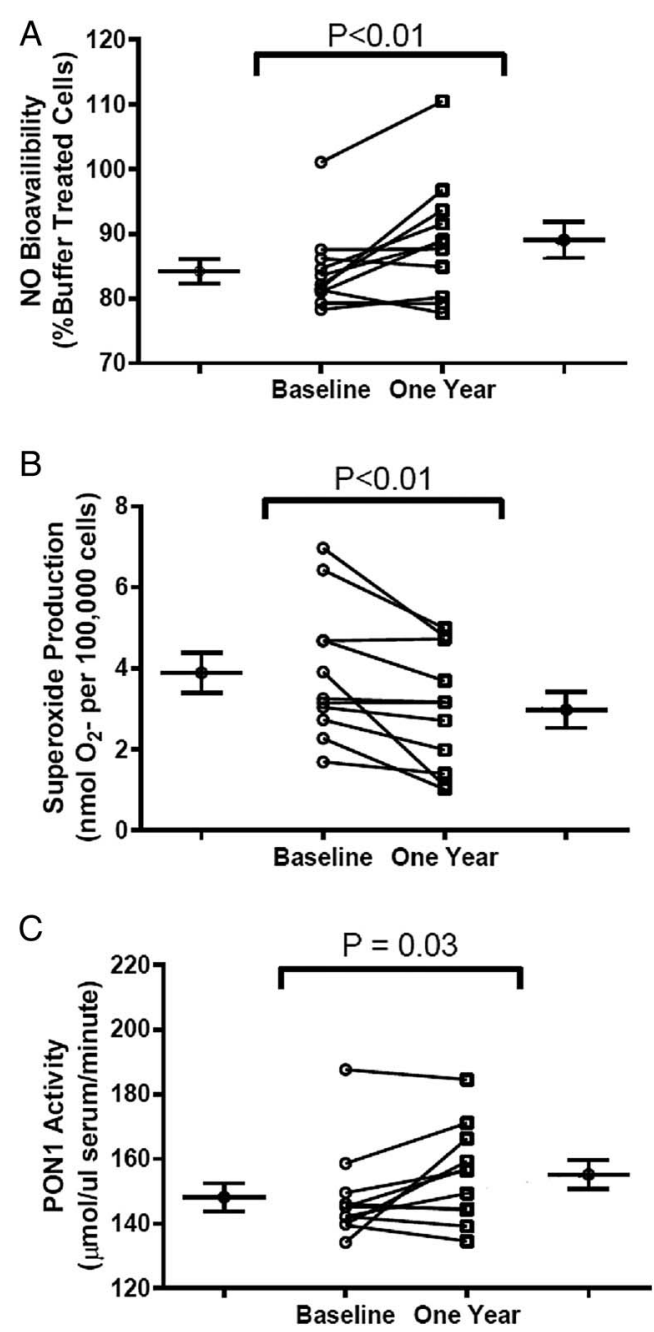

Figure 3 Trends in high-density lipoprotein function following 1 year infliximab treatment for (A) nitric oxide (NO) bioavailability, (B) superoxide production and (C) paraoxonase-1 (PON-1) activity. Individual data points for each patient $(n=18)$. Error bars represent mean and SEM. 
A

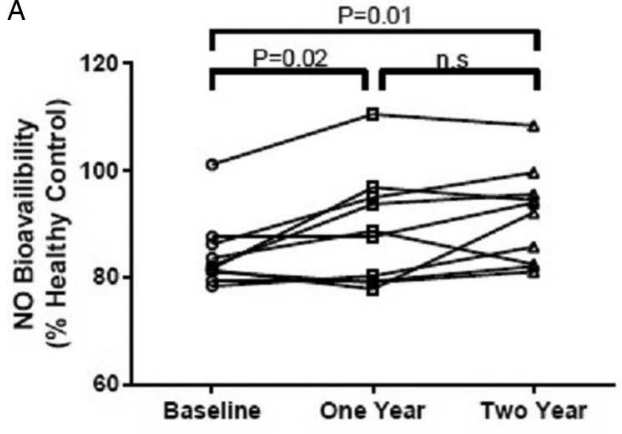

C

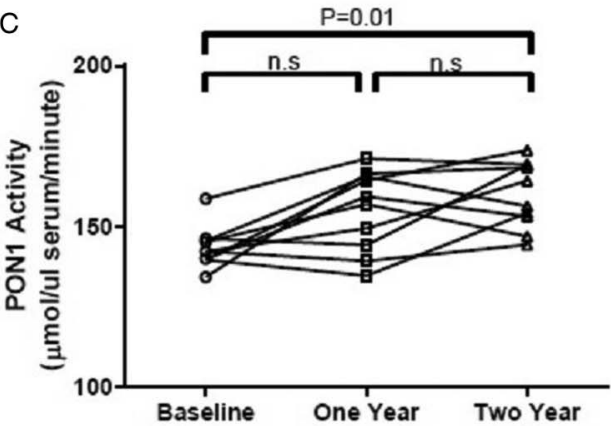

B

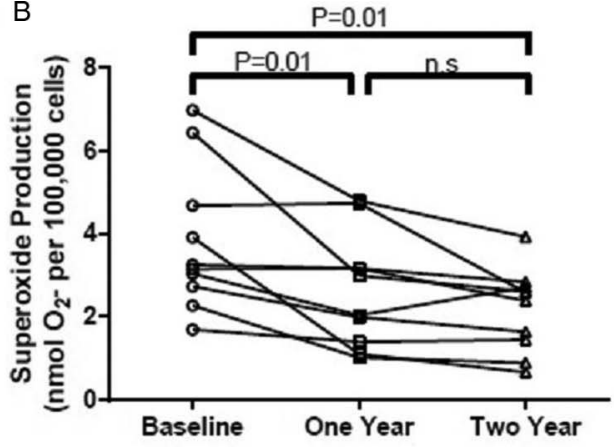

D

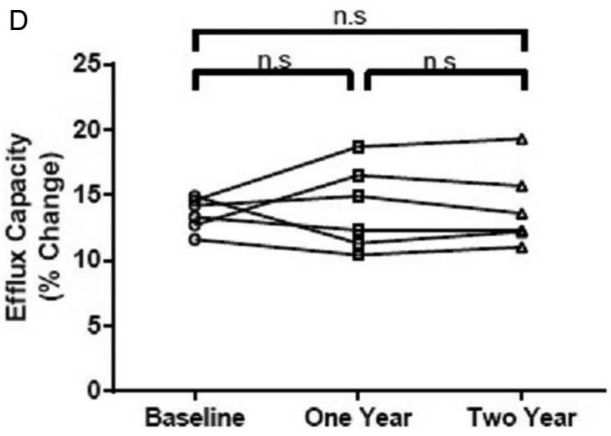

patients with erosive RA. These effects were not accompanied by changes in cholesterol efflux and tracked improvements in RA disease activity. Treatment with infliximab in addition to MTX for 2 years showed a benefit for a broad range of HDL functional properties including NO bioavailability, SO production and PON-1 activity. These findings suggest that there is a role for anti-inflammatory treatments, particularly those targeting the TNF- $\alpha$ pathway, for improving not only RA disease activity but also potentially CV risk factors in patients with RA.

The threefold increased risk of CV disease in patients with RA is not accounted for by traditional risk factors and is related to increased levels of inflammation. ${ }^{2}$ This influences a range of $\mathrm{CV}$ risk factors and causes quantitative and qualitative changes to lipoproteins. ${ }^{13}$ Substantial evidence has now accumulated, suggesting that inflammation can convert the normally protective HDL into a 'particle with adverse effects on endothelial function'. 414

We showed that HDL has several dysfunctional properties in patients with RA, which may contribute to their increased CV risk. Our findings are in agreement with previous evidence describing a pro-inflammatory HDL particle with reduced PON-1 activity. ${ }^{15}$ We have now demonstrated an impairment of the beneficial effects of HDL on endothelium with a marked decrease in the stimulation of endothelial $\mathrm{NO}$ and increase in SO production compared with healthy controls. Diminished production of NO may reflect endothelial dysfunction, an early step in atherogenesis, and represents a novel pathway by which HDL dysfunction can promote arterial disease in patients with RA.

We evaluated the potential for HDL function to be restored following treatment with RA anti-inflammatory therapies. MTX is commonly used as a first-line treatment in early RA. Observational studies have demonstrated reduced rates of myocardial infarction and stroke with MTX therapy and the ongoing CIRT trial is evaluating the drug in secondary CV prevention. ${ }^{16} 17$ Its exact anti-inflammatory mechanisms remain uncertain, although it has been shown to mediate increased extracellular accumulation of adenosine around connective tissue and reduce levels of CRP and interleukin- $6 .{ }^{17}$ Biological inhibition of TNF- $\alpha$ with infliximab in addition to MTX is a more effective approach for reducing RA disease activity. TNF- $\alpha$ is a proximal cytokine and its blockade has well-established antiinflammatory actions and effects on lipid metabolism. ${ }^{18}$ At 54 weeks, NO bioavailability was significantly improved in patients who received infliximab, but not with MTX. Infliximab has previously been associated with increased AKT/eNOS phosphorylation and improved endothelial function. ${ }^{19} 20$ Our study provides a novel mechanism by which infliximab can improve endothelial function and contribute to vascular protection by TNF- $\alpha$ inhibition.

After 1 year of our double-blinded randomised trial, SO production was significantly reduced in both arms. MTX is known to inhibit several inflammatory and oxidative stress mediators, associated with increased lipid peroxidation. ${ }^{21}$ Oxidative modification of the HDL particle alters its protective phenotype. $^{22}$ Combination therapy with infliximab caused an additional reduction of SO production compared with MTX alone. Previous studies of infliximab in humans suggest that TNF- $\alpha$ inhibition protects against oxidative DNA damage and lipid peroxidation, and this may explain its additional efficacy in reducing SO production. ${ }^{23}$ Despite a trend towards an increased benefit of infliximab therapy at 1 year, between-treatment comparisons did not reach statistical significance.

Assay of PON-1 provided an additional measure of the antioxidative function of HDL but no significant difference was found in the double-blinded randomised study in either arm at 1 year possibly due to the small sample size. However, PON-1 activity was improved in the single-blinded study at 1 and 2 years treatment of infliximab. While the effect of MTX on PON-1 activity has not previously been reported, infliximab is known to improve PON-1 activity through the reduction of 
lipid peroxidation including myeloperoxidase (MPO) pathways. ${ }^{24}$ Site-specific oxidative modification of PON-1 by MPO impairs the enzymes ability to bind with APOA-1 and reduced MPO could explain the increased PON-1 activity in our patients. $^{25}$

We did not demonstrate any differences in cholesterol efflux with either treatment regimen. This suggests that antiinflammatory therapies have a more pronounced impact on the vascular properties of HDL than on its efflux capacity. These findings support previous reports from our group and indicate that the endothelial function of HDL may be more sensitive to modification than cholesterol efflux. ${ }^{6}$ However, antiinflammatory treatments including MTX and infliximab have recently been associated with improved cholesterol efflux in patients with RA, and associated with CV disease in both healthy and at-risk populations. ${ }^{10}$ 26-28 These differences may be due to methodological issues in the efflux measurement and their interpretation, and require further investigation.

Our study was not powered to assess the quantitative relationship between HDL function, inflammation and disease activity. This also limited our ability to test for between-treatment differences in the randomised study design, although combination therapy demonstrated a clear benefit in the single-blinded study. However, we have demonstrated that a range of vascular properties of HDL can be improved with anti-inflammatory drug therapy. HDL function did not return to normal levels following treatment, and this is likely to be as a result of the residual levels of inflammation even after 2 years treatment (data not shown).

The ability of anti-inflammatory treatments to modify CV risk remains an intense area of clinical research. Currently, three anti-inflammatory agents are the subject of phase III trials; MTX (CIRT), Darapladib (SOLID-TMI and STABILITY) and canakinumab (CANTOS). Recently, both the STABILITY and SOLID-TMI trials reported that darapladib failed to reduce the risk of $\mathrm{CV}$ death and myocardial infarction in primary and secondary prevention. ${ }^{29} 30$ The CIRT and CANTOS trials are due to report shortly, but our data suggest that monotherapy with MTX alone may not be sufficient. Inhibition of TNF- $\alpha$, a broad immune effector, is more likely to suppress inflammation and may be a better strategy for CV benefit.

Key messages

\section{What is already known on this subject?}

Clinical trials aimed at raising high-density lipoprotein (HDL)-cholesterol have thus far failed to improve cardiovascular (CV) outcome, and this may be explained by a dysfunctional HDL phenotype in disease.

\section{What might this study add?}

This study underlines the need to measure and better understand HDL function. It provides evidence for the use of anti-inflammatory treatments, particularly those modulating the tumour necrosis factor- $\alpha$ pathway, to restore the beneficial effects of HDL on the vasculature.

\section{How might this impact on clinical practice?}

Our findings are of relevance to many recent clinical trials evaluating both therapies aiming to raise HDL levels and anti-inflammatory agents with the aim of reducing CV risk.
This study underlines the need to measure and better understand HDL function. It provides evidence for the use of antiinflammatory treatments, particularly those modulating the TNF- $\alpha$ pathway, to restore the beneficial effects of HDL on the vasculature. Our findings are of relevance to many recent clinical trials evaluating both HDL elevation and anti-inflammatory therapies with the aim of reducing CV risk.

\section{Author affiliations}

${ }^{1}$ Vascular Physiology Unit, Institute of Cardiovascular Science, University College London, London, UK

${ }^{2}$ Institute of Structural \& Molecular Biology and London Centre for Nanotechnology, University College London, London, UK

${ }^{3}$ Periodontology Unit, Department of Clinical Research, University College London Eastman Dental Institute, London, UK

${ }^{4}$ Department of Cardiology, Charite Universitätsmedizin Berlin, Berlin, Germany ${ }^{5}$ Kennedy Institute of Rheumatology, University of Oxford, Oxford, UK

${ }^{6}$ National Institute for Cardiovascular Outcomes Research, University College London, London, UK

Contributors FON, MC, PC and JD made substantial contributions to the conception and design of the work. FON, EM, NP and ES made substantial contributions to the acquisition, analysis or interpretation of data for the work. FON, MC, CWMK, UL, FD, PCT and JD made substantial contribution to the work or revising it critically for important intellectual content and final approval of the version to be published.

Funding F. Hoffmann-La Roche and the Leducq Foundation.

Competing interests None declared.

\section{Patient consent Obtained.}

Ethics approval Riverside Research Ethics Committee.

Provenance and peer review Not commissioned; externally peer reviewed.

Open Access This is an Open Access article distributed in accordance with the Creative Commons Attribution Non Commercial (CC BY-NC 4.0) license, which permits others to distribute, remix, adapt, build upon this work non-commercially, and license their derivative works on different terms, provided the original work is properly cited and the use is non-commercial. See: http://creativecommons.org/ licenses/by-nc/4.0/

\section{REFERENCES}

1 Meune C, Touzé E, Trinquart L, et al. Trends in cardiovascular mortality in patients with rheumatoid arthritis over 50 years: a systematic review and meta-analysis of cohort studies. Rheumatology (Oxford) 2009;48:1309-13.

2 del Rincón ID, Williams K, Stern MP, et al. High incidence of cardiovascular events in a rheumatoid arthritis cohort not explained by traditional cardiac risk factors. Arthritis Rheum 2001;44:2737-45.

3 Myasoedova E, Crowson CS, Kremers HM, et al. Lipid paradox in rheumatoid arthritis: the impact of serum lipid measures and systemic inflammation on the risk of cardiovascular disease. Ann Rheum Dis 2011;70:482-7.

4 Charakida M, Besler C, Batuca JR, et al. Vascular abnormalities, paraoxonase activity, and dysfunctional $\mathrm{HDL}$ in primary antiphospholipid syndrome. JAMA 2009:302:1210-17.

5 McMahon M, Grossman J, FitzGerald J, et al. Proinflammatory high-density lipoprotein as a biomarker for atherosclerosis in patients with systemic lupus erythematosus and rheumatoid arthritis. Arthritis Rheum 2006;54:2541-9.

6 O'Neill FP, Riwanto M, Charakida M, et al. Structural and functional changes in HDL with low grade and chronic inflammation. Int J Cardiol 2015;188:111-16.

7 Taylor PC, Steuer A, Gruber J, et al. Ultrasonographic and radiographic results from a two-year controlled trial of immediate or one-year-delayed addition of infliximab to ongoing methotrexate therapy in patients with erosive early rheumatoid arthritis. Arthritis Rheum 2006;54:47-53.

8 Tong H, Knapp HR, VanRollins M. A low temperature flotation method to rapidly isolate lipoproteins from plasma. J Lipid Res 1998;39:1696-704.

9 Besler $C$, Heinrich K, Rohrer $L$, et al. Mechanisms underlying adverse effects of HDL on eNOS-activating pathways in patients with coronary artery disease. J Clin Invest 2011;121:2693-708.

10 Khera AV, Cuchel M, de la Llera-Moya M, et al. Cholesterol efflux capacity, highdensity lipoprotein function, and atherosclerosis. N Engl J Med 2011;364:127-35.

11 Bhattacharyya T, Nicholls SJ, Topol EJ, et al. Relationship of paraoxonase 1 (PON1) gene polymorphisms and functional activity with systemic oxidative stress and cardiovascular risk. JAMA 2008;299:1265-76.

12 O'Neill F, McLoughlin E, Riwanto M, et al. Reproducibility and biological variability of HDL's vascular functional assays. Atherosclerosis 2015;241:588-94. 
13 González-Gay MA, González-Juanatey C. Inflammation and lipid profile in rheumatoid arthritis: bridging an apparent paradox. Ann Rheum Dis 2014; 73:1281-3.

14 Hahn BH, Lourencço EV, McMahon M, et al. Pro-inflammatory high-density lipoproteins and atherosclerosis are induced in lupus-prone mice by a high-fat diet and leptin. Lupus 2010;19:913-17.

15 Popa C, van Tits LJ, Barrera P, et al. Anti-inflammatory therapy with tumour necrosis factor alpha inhibitors improves high-density lipoprotein cholesterol antioxidative capacity in rheumatoid arthritis patients. Ann Rheum Dis 2009;68:868-72.

16 Everett BM, Pradhan AD, Solomon $\mathrm{DH}$, et al. Rationale and design of the Cardiovascular Inflammation Reduction Trial: a test of the inflammatory hypothesis of atherothrombosis. Am Heart J 2013;166:199-207.

17 Westlake SL, Colebatch AN, Baird J, et al. The effect of methotrexate on cardiovascular disease in patients with rheumatoid arthritis: a systematic literature review. Rheumatology (Oxford) 2010;49:295-307.

18 Tracey D, Klareskog L, Sasso EH, et al. Tumor necrosis factor antagonist mechanisms of action: a comprehensive review. Pharmacol Ther 2008;117: 244-79.

19 Filho AG, Kinote A, Pereira DJ, et al. Infliximab prevents increased systolic blood pressure and upregulates the AKT/eNOS pathway in the aorta of spontaneously hypertensive rats. Eur J Pharmacol 2013;700: 201-9.

20 Hürlimann D, Forster A, Noll G, et al. Anti-tumor necrosis factor-alpha treatment improves endothelial function in patients with rheumatoid arthritis. Circulation 2002:106:2184-7.
21 Wessels JA, Huizinga TW, Guchelaar HJ. Recent insights in the pharmacological actions of methotrexate in the treatment of rheumatoid arthritis. Rheumatology (Oxford) 2008;47:249-55.

22 Undurti A, Huang Y, Lupica JA, et al. Modification of high density lipoprotein by myeloperoxidase generates a pro-inflammatory particle. J Biol Chem 2009:284:30825-35.

23 Kageyama Y, Takahashi M, Ichikawa $T$, et al. Reduction of oxidative stress marker levels by anti-TNF-alpha antibody, infliximab, in patients with rheumatoid arthritis. Clin Exp Rheumatol 2008;26:73-80.

24 Bacchetti T, Campanati A, Ferretti G, et al. Oxidative stress and psoriasis: the effect of antitumour necrosis factor- $\alpha$ inhibitor treatment. Br J Dermatol 2013;168:984-9.

25 Huang $Y$, Wu Z, Riwanto $M$, et al. Myeloperoxidase, paraoxonase-1, and HDL form a functional ternary complex. J Clin Invest 2013;123:3815-28.

26 Voloshyna I, Seshadri S, Anwar K, et al. Infliximab reverses suppression of cholesterol efflux proteins by TNF- $\alpha$ : a possible mechanism for modulation of atherogenesis. Biomed Res Int 2014;2014:312647.

27 Rohatgi A, Khera A, Berry JD, et al. HDL cholesterol efflux capacity and incident cardiovascular events. N Engl J Med 2014;371:2383-93.

28 Ronda N, Greco D, Adorni MP, et al. Newly identified antiatherosclerotic activity of methotrexate and adalimumab: complementary effects on lipoprotein function and macrophage cholesterol metabolism. N Engl J Med 2015;67:1155-64.

29 White HD, Held C, Stewart R, et al. Darapladib for preventing ischemic events in stable coronary heart disease. N Engl J Med 2014;370:1702-11.

30 O'Donoghue ML, Braunwald E, White HD, et al. Effect of darapladib on major coronary events after an acute coronary syndrome: the SOLID-TIMI 52 randomized clinical trial. JAMA 2014;312:1006-15. 